Conclusion In summary, we show that the PelvoCheck ${ }^{\circledR}$ CT/ NG assay is a highly sensitive and highly specific method for the detection of CT and NG. To our knowledge, this is the first commercial CT/NG test system validated for the analysis of pooled urine specimens.

\section{P2.018 CRYPTOSPORIDIOSIS AND CYCLOSPORIDIASIS, TWO MAJOR INFECTIONS IN HIV POSITIVE PATIENTS, IN BUKAVU, D.R.CONGO}

doi:10.1136/sextrans-2013-051184.0283

'N A Bulabula, ${ }^{1} \mathrm{~A}$ B Shindano, ${ }^{2} \mathrm{~A}$ M Bulabula, ${ }^{3} \mathrm{~J}$ B Nachega, ${ }^{4} \mathrm{R}$ Fiasse. ${ }^{1}$ Provincial General Hospital of Bukavu, Bukavu, Congo; '2Universite de Kindu, Kindu, Congo; '3Johns Hopkins Bloomberg School of Public Health, Baltimore, MD, United States; ${ }^{4}$ Universite Catholique de Louvain, Bruxelles, Belgium

Background There are little data on the prevalence and clinical outcomes of coccidian parasites infection in HIV positive patients in Africa.

Methods We conducted a cross-sectional study at the Opportunistic Infections Clinic of the Provincial Hospital of Bukavu, from April 2010 to October 2011. HIV patients attending the clinic, aged 15 years and above were included after obtaining their consent, the CD4 count and patient details were recorded, stool samples were collected according to the WHO procedure. A single stool sample was collected and conserved in formalin - ether $10 \%$ and transported to the Provincial Laboratory of Bukavu for analysis. A consecutive sample of 108 patients was collected.

Smears were stained according to the modified ziehl neelsen procedure and examined at x100 magnification using an oil immersion microscope. Data were analysed with EPI-INFO 3.5. Differences with P-values $<0.05$ were considered significant.

Results $58.3 \%$ were female. Mean $( \pm$ SD) age $40 \pm 15.7$ years old. Stools were diarrheic in $66.7 \%$. Overall prevalence of Cryptosporidium spp was $57.9 \%$ (95\% CI: 41.8 - 76.1), Cyclospora cayetanensis $31.6 \%$ (95\% CI: 19.3 - 47.5). 63.9\% of patients were on antiretroviral therapy. Patients with CD4 counts $<50$ cells $/ \mu \mathrm{L}$, presented with a higher prevalence of either Cryptosporidium spp $(62.1 \%)$ or Cyclospora cayetanensis $(27.6 \%)(\mathrm{P}=0.0000)$. In diarrheic stools we recorded 59.6\% (28/47) Cryptosporidium spp $(\mathrm{P}=0.0001), 27.7 \%$ (13/47) Cyclospora cayetanensis. Dual infections were more noted in the CD4 count range of $<100$ cells $/ \mu \mathrm{L}$, $63.4 \%(26 / 41)$ for CD4 count $<50$ cells $/ \mu \mathrm{L}$ and $36.6 \%(15 / 41)$ for CD4 $50-100$ cells $/ \mu \mathrm{L}(\mathrm{P}=0.0000)$. On ARV, only subjects with100 - 200 cells $/ \mu \mathrm{L}$ of CD4, had a good clinical evolution $(\mathrm{P}=0.0000)$

Conclusions Cryptosporidium parvum and Cyclospora cayetanensis are highly prevalent in HIV patients with advanced diseases and major causes of chronic diarrhoea. Dual infection is related to very low CD4 count.

\section{P2.019 ANALYTICAL SPECIFICITY AND SENSITIVITY OF THE APTIMA COMBO 2 AND APTIMA GC ASSAYS FOR DETECTION OF NEISSERIA GONORRHOEAE ON THE GEN- PROBE PANTHER INSTRUMENT AND VERIFICATION OF SPECIMENS POSITIVE FOR $\boldsymbol{N}$. GONORRHOEAE USING OTHER COMMERCIAL DIAGNOSTIC NAATS}

doi:10.1136/sextrans-2013-051184.0284

'D Golparian, ${ }^{2} \mathrm{~S}$ Tabrizi, ${ }^{1} \mathrm{~S}$ Jacobsson, ${ }^{3} \mathrm{C}$ Stezckó Nilsson, ${ }^{1} \mathrm{H}$ Fredlund, 'M Unemo. ${ }^{1} \mathrm{WHO}$ Collaborating Centre for Gonorrhoea and other STIs, Department of Laboratory Medicine, Microbiology, Örebro University Hospital, ÖREBRO, Sweden; '2Department of Microbiology and Infectious Diseases, the Royal Women's Hospital, Parkville, Australia; ${ }^{3}$ Department of Dermatology and Venereology, Örebro University Hospital, ÖREBRO, Sweden

Objectives Nucleic acid amplification tests (NAATs) have rapidly replaced culture for the detection of Neisseria gonorrhoeae in many countries worldwide. Several commercial gonococcal NAATs have received US FDA clearance, including the APTIMA Combo 2 assay (AC2) and APTIMA GC assay (AGC) (Gen-Probe, San Diego, CA USA). In this study, the analytical specificity and sensitivity of AC2 and AGC were evaluated on the Gen-Probe PANTHER instrument and specimens positive for $N$. gonorrhoeae using commercial diagnostic NAATs were verified by AGC.

Methods Samples spiked with 503 bacterial isolates (298 non-gonococcal Neisseria isolates and 205 gonococci) were tested. All initially equivocal and false-positive/false-negative results were verified according to a strict algorithm for confirmatory testing. Furthermore, 92 selected specimens tested positive for $N$. gonorrhoeae on Abbott RealTime PCR CT/NG (Abbott Laboratories) $(n=19)$, COBAS 4800 CT/ NG (Roche Molecular Systems Inc.) ( $\mathrm{n}=34$ ), or BD ProbeTec ET/Ox Chlamydia trachomatis and Neisseria gonorrhoeae Amplified DNA (Becton Dickinson) assays $(\mathrm{n}=39$ ) were examined for confirmation with AGC. For discrepancy analysis, a gonococcal diagnostic duplex PCR (targeting the porA pseudogene and opa genes) was used.

Results Both AC2 and AGC had 100\% analytical specificity and sensitivity. Moreover, the verification of positive specimens from other commercial NAATs showed that all $(100 \% ; 19 / 19)$ specimens from Abbott RealTime PCR CT/NG, 94\% (32/34) from COBAS 4800 CT/NG, and 51\% (20/39) from BD ProbeTec ET/Ox Chlamydia trachomatis and Neisseria gonorrhoeae Amplified DNA could be verified as true positive in AGC (AGC results were confirmed in the gonococcal duplex PCR)

Conclusion The analytical specificity and sensitivity of AC2 and AGC were substantially challenged, and both assays displayed $100 \%$ specificity and sensitivity. This study also shows that AGC can be used for confirmatory testing as well as emphasises the importance of verifying particularly $N$. gonorrhoeae specimens that are low-positive or from extragenital sites with an alternative NAAT target.

\section{P2.020 DIAGNOSTIC ACCURACY OF RAPID TESTS FOR C. TRACHOMATIS, N. GONORRHOEA AND SYPHILIS AT THE POINT OF CARE IN WOMEN WITH SYMPTOMS OF LOWER GENITAL TRACT INFECTION}

doi:10.1136/sextrans-2013-051184.0285

${ }^{1} \mathrm{~L}$ Nuñez-Forero, ${ }^{1} \mathrm{~L}$ Moyano, ${ }^{2} \mathrm{E}$ Angel Muller, ${ }^{3} \mathrm{~A}$ Rodriguez, ${ }^{3} \mathrm{~A}$ Ruiz Parra, ${ }^{4} \mathrm{~J}$ Tolosa, ${ }^{3}$ H Gaitan Duarte. ${ }^{1}$ Health Secretariat, Bogota, Colombia; ${ }^{2}$ Department of Obstetrics and Gynecology, Universidad Nacional de Colombia, Bogota, Colombia; ${ }^{3}$ Clinical Research Institute, Universidad Nacional de Colombia, Bogota, Colombia; ${ }^{4}$ Oregon and Health Science University., Portland, OR, United States

Objective Evaluate the diagnostic accuracy of rapid tests (RTs) for C. trachomatis (CT), N. gonorrheae (NG), and syphilis at the point of care for management of women with symptoms of lower genital tract infection (LGTI).

Materials and Methods: Diagnostic validity study assembled in a cohort study of sexually active women 14 to 49 years old, consulting with symptoms of LGTI at three outpatient facilities in Bogotá, Colombia in 2010, after ethics board approval. Exclusion criteria: hysterectomy, pregnancy, or receiving antibiotics in the 7 previous days. Sampling: sequential. RTs evaluated: Acon ${ }^{\circledR}$ duo and Acon plate $^{\circledast}$ (Acon, San Diego, CA, USA) for NG. Acon ${ }^{\circledast}$ Plate, Acon Duo and QuickVue ${ }^{\circledR}$ (Quidel Corporation, San Diego, CA, USA). for CT They were compared against polymerase chain reaction (PCR) AMPLICOR C.trachomatis/N. gonorrhoeae test (Roche Diagnostic Systems, Inc., Branchburg, N.J.). Bioline ${ }^{\circledast}$ Syphilis 3.0 (Standard Diagnostics, Inc., Kyunggi-do, South Korea) and ACON ${ }^{\circledR}$ Syphilis for syphilis and were compared to a positive RPR and TPHA. Sensitivity $(S)$, specificity $(\mathrm{Sp})$, positive and negative likelihood ratios LR $(+)$, LR (-) were calculated.

Results 1410 subjects recruited. The prevalence of NG and CT with PCR was 1.4\% (19/1376) and 9.6\% (133/1379) respectively. 